\title{
A study for 2-D indoor localization using multiple leaky coaxial cables
}

\author{
JUNJIE ZHU, ${ }^{1}$ (1) PENGCHENG HOU, ${ }^{1}$ YAFEI HOU, ${ }^{1}$ (1) SATOSHI DENNO ${ }^{1}$ AND MINORU OKADA ${ }^{2}$
}

\begin{abstract}
Indoor localization technology, which can provide the location information of the target object or stochastic things, is becoming essential requirement for many applications and services such as Internet-of-Things (IoT), real-time control in the development of Fifth-generation $(5 G)$ technology. Leaky coaxial cable which can be used as antennas is able to detect the location of the user in a simple way due to its potential property. In this paper, we proposes a simple method to improve the localization accuracy of 2-D indoor localization using multiple LCX cables. In addition, we also evaluate the channel capacity loss due to the localization error of the LCX-MIMO using our proposed method.
\end{abstract}

Keywords: Leaky coaxial cable, Capacity, MIMO

Received 30 June 2020; Revised 25 August 2020

\section{INTRODUCTION}

Fifth-generation $(5 \mathrm{G})$ technologies provide the possibility for the realization of networking vast numbers of mobile and stationary devices, vehicles, user terminals, sensors, and robots. These network interactions and communications support the development of the Internet-of-Things (IoT) [1] and real-time dynamic networks. Positioning technology which provides the accurate location information of the target object or stochastic things is becoming the necessary and vital requirement for many applications and services such as autonomous vehicles [2], health care [3], precision agriculture [4], industrial and manufacturing automation, and robotics [5]. All these applications with location-aware technologies will significantly promote the penetration of $5 \mathrm{G}$ technology into our social life.

As the most commonly used positioning technology, global positioning system (GPS) provides navigation and localization service for almost global users. However, the GPS positioning is not effective in the indoor applications or harsh environments. Positioning technologies, which are used for indoor environment without the requirement of GPS or direct access to base station, have been researched for years and can be roughly divided into two categories. One is based on the analysis of the propagation characteristics of the signal radio wave to estimate the location

\footnotetext{
${ }^{1}$ Graduate School of Natural Science and Technology, Okayama University, Okayama City, Okayama, 700-8530, Japan

${ }^{2}$ Division of Information Science, Nara Institute of Science and Technology, Nara, 630-0192, Japan

Corresponding author:

Yafei Hou

Email: yfhou@okayama-u.ac.jp
}

information. The most commonly used methods include angle of arrival (AoA), time of arrival (ToA), time difference of arrival (TDoA), channel state information (CSI), and received signal strength (RSS) [6-10]. The other category is the methods based on other medium and examples include visible light [11], infrared [12], ultrasonic [13], camera-imaging [14], etc. These methods provide more solutions for positioning but need to add additional sensors to the transmitter side or the receiver side with additional power and cost requirements.

Leaky coaxial cable (LCX) which can be used as antennas has been found that it can play an important role in positioning for diverse application scenarios. Researches on LCX have been for several years, and we find many potential advantages of LCX cable such as simple installation, uniform wireless coverage [15], low interference between cells, and available for wireless power transfer (WPT) [16]. In addition, LCX also can be designed and used for highfrequency bands in $[17,18]$, and [19] shows the employment of LCX for train and the train ground communication system. For positioning and location-awareness, compared with other commonly used antennas such as monopole antenna, LCX can easily detect the location of the user terminal in its wireless coverage area. Previous researches have developed several detection methods for LCX systems. Ref. [20] shows the method based on ToA for 1-D location detection using LCX cable. Here, one LCX cable is treated as only one antenna for usage. Researches in [21-24] show that LCX cable has bi-directional property and one LCX can be utilized as two antennas for 2-by-2 LCX multiple input multiple output (MIMO) system. In addition, a 4-by-4 LCX-MIMO system has been proposed in [25]. From that, a method based on TDoA using the time difference between 
the signal arrival at different ends of the LCX to detect the user location has been proposed in $[26,27]$. Other positioning methods such as designed pulse signal method are studied in $[28-31]$.

Most researches of LCX positioning usually use one LCX or two placed in parallel for 2-D location detection. As the positioning performance results in previous researches, we find that the location positioning accuracy of the direction perpendicular to LCX cable is worse than that of the direction parallel to the LCX cable. Based on this fact, in this paper, we propose a LCX positioning method using two LCXs perpendicular to each other to improve the positioning accuracy of the LCX system. The results show that the positioning performance integrating both location information from two LCXs perpendicular to each other is better than that of conventional method. On the other hand, a low-complexity power allocation method using user's position information for LCX-MIMO is proposed in [32]. This paper will also evaluate the capacity performance of the LCX-MIMO with the proposed positioning method.

The rest of paper is structured as follows. In Section II, we introduce the structure and the propagation property of LCX cable. Then the proposed LCX positioning method will be shown in Section III. We introduce the concept of MIMO channel capacity with power allocation methods in Section IV. The experiment and the comparison results of positioning and capacity performance will be shown in Section V. The paper will be ended with simple conclusion in Section VI.

\section{LCX STRUCTURE AND RADIATION PRDPERTY}

The LCX cable used for wireless communication usually has a four-layer structure as Fig. 1 shows. As the characteristic of the LCX, the outer conductor has many slots placed at equal distances and radio wave will be received and radiated from these slots. We assume that the plane perpendicular to the traveling direction of the radio wave propagating through the LCX is o degree, the radiation angle of the radio wave $\theta_{m}$ can be given as

$$
\theta_{m}=\sin ^{-1}\left(\sqrt{\epsilon_{r}}+\frac{m \lambda}{P}\right), \quad(m=-1,-2, \ldots)
$$

where $m$ is the harmonic order, $P$ is the period of slots, and $\epsilon_{r}$ is the LCX's relative insulator permittivity. $\lambda$ is the wavelength related to the frequency band. $m$ is set as -1 to avoid radiated harmonics. It is possible to change the main radiation angle of the LCX cable by adjusting the direction of the slot and the value of $P$.

Figure 2 shows the bidirectional radiation property of the LCX when inputting signals to both side of the cable without the terminating resistor [21]. Two different peak directivities are obtained and the crossing angle is about $2 \theta_{-1}$ degree, their radiation characteristics have low correlation with each other. 2-by-2 and 4-by-4 LCX-MIMO are proposed with this radiation property [25].

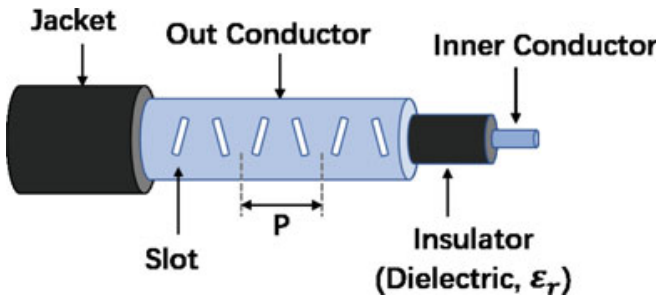

Fig. 1. The structure of LCX cable.

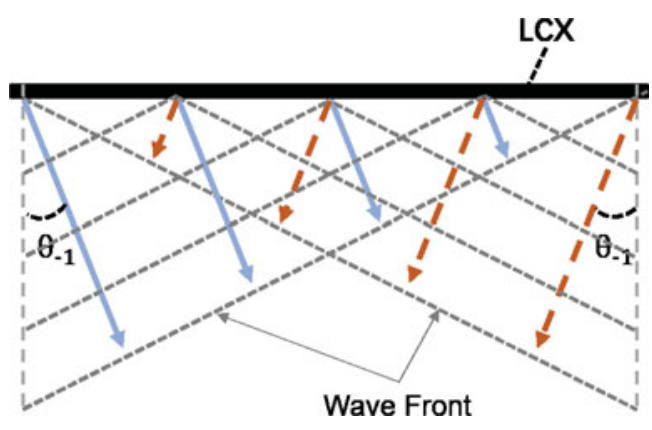

Fig. 2. Bi-directional radiation property of LCX.

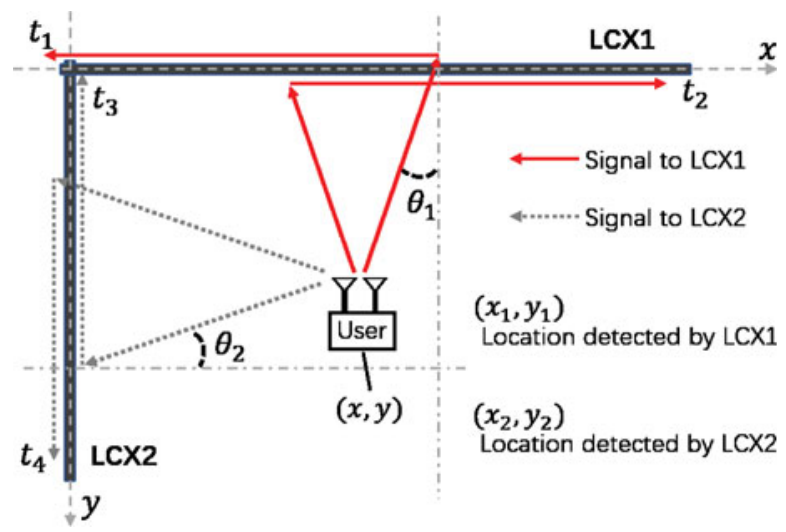

Fig. 3. Proposed LCX-MIMO system.

\section{PRDPDSED LCX SYSTEM AND POSITIONING METHOD}

\section{A) Proposed LCX-MIMO system}

Figure 3 shows the LCX-MIMO system proposed in this paper. Different from placing the LCXs in parallel in previous research, two LCXs with maximum radiation angles of $\theta_{1}, \theta_{2}$ are set perpendicular to each other. The main idea of the method is based on the fact that the location positioning accuracy of the direction perpendicular to the LCX cable is worse than that of the direction parallel to the LCX cable [27]. The proposal adds an additional LCX to improve detection accuracy of single LCX in the vertical direction.

We set the $X$-axis as the direction along the LCX1 and the $Y$-axis as the direction along the LCX2. The mobile user is arranged in the overlapping wireless coverage area of the two LCXs. It is assumed that the propagation path of the signal radio wave in the space is a straight line when the signals transmitted from the user terminal reach both 
ends of each LCX. We use the LCX1 as the example to show the relationship between signal arrival time, radiation angle, and location coordinate of the user terminal. The path of the signal from the user to one end of the LCX1 include two parts: propagation in the air and propagation in the cable. Therefore, the arrival times can be calculated as

$$
\begin{gathered}
t_{1}=\frac{y_{1}}{c \cos \theta_{1}}+\frac{x_{1}+y_{1} \tan \theta_{1}}{v_{1} c}, \\
t_{2}=\frac{y_{1}}{c \cos \theta_{1}}+\frac{L_{1}-x_{1}+y_{1} \tan \theta_{1}}{v_{1} c},
\end{gathered}
$$

where $v_{1}$ is the shortening coefficient of signal wavelength in LCX 1 and $c$ is the speed of light. $L_{1}$ is the length of the LCX1. Then the location of the user over $X$-axis can be calculated as

$$
x_{1}=\frac{\left|t_{1}-t_{2}\right| v_{1} c+L_{1}}{2} .
$$

Then, we can calculate the location over $Y$-axis using $x_{1}$, arrival time $t_{2}$, and radiation angle $\theta_{1}$ as

$$
y_{1}=\frac{\left(c t_{2}-\left(\left(L_{1}-x_{1}\right) / v_{1}\right)\right) v_{1} \cos \theta_{1}}{v_{1}+\sin \theta_{1}} .
$$

$\left(x_{1}, y_{1}\right)$ is the location detected by LCX 1 and with the same process we can estimate the location detected by LCX 2 as

$$
\begin{gathered}
y_{2}=\frac{\left|t_{3}-t_{4}\right| v_{2} c+L_{2}}{2}, \\
x_{2}=\frac{\left(c t_{4}-\left(\left(L_{2}-y_{2}\right) / v_{2}\right)\right) v_{2} \cos \theta_{2}}{v_{2}+\sin \theta_{2}},
\end{gathered}
$$

where $v_{2}$ is the shortening coefficient of signal wavelength in LCX2. $L_{2}$ is the cable length. It should be noted that in the positioning process of the LCX2 placed vertically, the $Y$-axis coordinate $y_{2}$ is first calculated from the time difference of arrival, and then the $X$-axis coordinate $x_{2}$ is calculated. Then we can get the $y$ coordinate as

$$
y=\frac{y_{1}+y_{2}}{2}
$$

and the $x$ can be calculated by

$$
x=\frac{L_{1}-\left(c t_{2}-\frac{y\left(v_{1}+\sin \theta_{1}\right)}{v_{1} \cos \theta_{1}} v_{1}\right)+x_{1}}{2} .
$$

\section{B) Arrival time estimation by MUSIC method}

The MUtiple SIgnal Classification (MUSIC) method is a relatively simple and efficient eigenstructure method of DOA estimation. The method estimates the noise subspace from available samples and searches for steering vectors that are as orthogonal to the noise subspace as possible. This is normally accomplished by searching for peaks in the MUSIC spectrum.

In this paper, we use the MUSIC method to estimate the arrival time of the signal from user terminal to cable end. Here, we assume the receiving signal, $x(n)$, consisting of $s$ complex exponentials in the presence of Gaussian white noise to be

$$
x(n)=\sum_{i=1}^{s} A_{i} e^{-j 2 \pi f_{i} \tau}+w(n)
$$

where $A_{i}$ is the amplitude of the signal and $f_{i}$ is the frequency. $w(n)$ is the Gaussian white noise. Given an $M \times$ $M$ autocorrelation matrix as

$$
\mathbf{R x}=\sum_{i=1}^{M} d_{i} \mathbf{u}_{i} \mathbf{u}_{i}^{H}
$$

the $d_{i}$ is the eigenvalue, $\mathbf{u}_{i}$ is the eigenvector corresponding to the eigenvalue. We use the superscript $H$ to represent the Hermitian transpose. If the eigenvalues are sorted in decreasing order, the eigenvectors corresponding to the $s$ largest eigenvalues span the signal subspace. The remaining $M-s$ eigenvectors span the orthogonal space, where there is only noise. Therefore, the matrix $\mathbf{R}_{x}$ can be composed of two parts as

$$
\mathbf{R x}=\sum_{i=1}^{s}\left(\lambda_{i}+\sigma_{w}^{2}\right) \mathbf{u}_{i} \mathbf{u}_{i}^{H}+\sum_{i=s+1}^{M} \sigma_{w}^{2} \mathbf{u}_{i} \mathbf{u}_{i}^{H} .
$$

Here, $\lambda_{i}$ is the eigenvalue of the correlation matrix consisting of signal only and $\sigma_{w}$ is the variance of white noise. The estimation function for MUSIC can be defined as

$$
g(\tau)=\frac{1}{\sum_{i=s+1}^{M}\left|\mathbf{u}_{i}^{H} \mathbf{e}(\tau)\right|^{2}},
$$

where $\mathbf{e}(\tau)$ is known as the steering vector and can be expressed as

$$
\begin{gathered}
\mathbf{e}(\tau)=\left[1, e^{-j 2 \pi f_{1} \tau}, e^{-j 2 \pi f_{2} \tau}, \ldots, e^{-j 2 \pi f_{M} \tau}\right]^{T}, \\
\tau=n T_{r},\left(n=0,1,2, \ldots, N_{s}-1\right) .
\end{gathered}
$$

$T_{r}$ is the time resolution of MUSIC method. $N_{s}$ is the number of elements in pseudo spectrum, and $N_{S} T_{r}$ is the maximum observation time for signal arrival. The orthogonality between the noise subspace and the steering vectors will minimize the denominator equation (13) to o value. However, it is a small value in practice because of the noise. As a result, it will give rise to peaks in $g(\tau)$ in equation (13). The largest peak corresponds to the signal arrival and then we can estimate the ToA.

IV. LCX-MIMO CHANNEL CAPACITY WITH LOCALIZATION ERRDR

\section{A) LCX-MIMO and channel capacity with power allocation method}

As introduced in the previous section, when signals are fed to both sides of the LCX cable, the intersection angle of the two radiation directions will be $2 \theta_{-1}$ degree. Their radiation 
characteristics have low correlation with each other and we can obtain a good 2-by-2 LCX-MIMO channel. In addition, we can realize a 4-by-4 LCX-MIMO channel by using two LCX cables with different radiation property.

MIMO channel capacity named as Shannon ergodic capacity shows a fundamental property and limitation of MIMO system [33]. The channel capacity can be described under two conditions. One is that the channel state information (CSI) is unknown for the transmitter side. It usually uses the equal power (EP) allocation method by allocating power to each stream equally to obtain promising channel capacity. The EP allocation method can be expressed as

$$
C_{E P}=\mathbb{E}_{\mathbf{H}}\left[\log _{2}\left(\operatorname{det}\left[\mathbf{I}+\frac{\zeta}{N} \mathbf{H H}^{H}\right]\right)\right] .
$$

Here, we assume that the CSI $\mathbf{H}$ is only known at receiver side and $\zeta$ is the average signal-to-noise ratio (SNR). $N$ and I are the antenna number at transmitter and the identity matrix. The superscript $H$ represents the Hermitian transpose. In the other condition, if the CSI is known, we can get the optimal capacity by water-filling (WF) algorithm method [34]. The channel capacity with WF method can be expressed as

$$
C_{W F}=\mathbb{E}_{\mathbf{H}}\left[\sum_{i=1}^{N} \log _{2}\left(1+\lambda_{i} P_{i}\right)\right]
$$

Here $\lambda_{i}$ is the corresponding eigenvalue of CSI matrix. $P_{i}$ is the power allocated for the $i$ th MIMO eigenchannel as

$$
P_{i}=\left(\mu-\frac{1}{\lambda_{i}}\right)^{+}
$$

where $x^{+}$means the result of $\max (x, 0)$ and $\mu$ is water-fill level. The WF algorithm fill each eigenchannel with power to the level $\mu$ iteratively and the MIMO channel capacity is represented as

$$
C_{W F}=\mathbb{E}_{\mathbf{H}}\left[\sum_{i=1}^{N}\left(\log _{2}\left(\lambda_{i} \mu\right)\right)^{+}\right]
$$

Equations (16) and (19) above are normalized by bandwidth.

\section{B) LCX-MIMO channel capacity with user location}

In addition to EP allocation method and WF allocation method, the research in [32] has proposed and evaluated a low-complexity transmission power allocation method for LCX-MIMO channel using the position information (PI) of the user. The results show that the proposed power allocation method using user PI can improve the channel capacity with almost the same low computational complexity as the EP method. The main idea of the method is adjusting the power over receiver side in consideration of the known constant power loss in the LCX cable. We use Fig. 4 to show the method, user $U$ with two antennas is set in the wireless

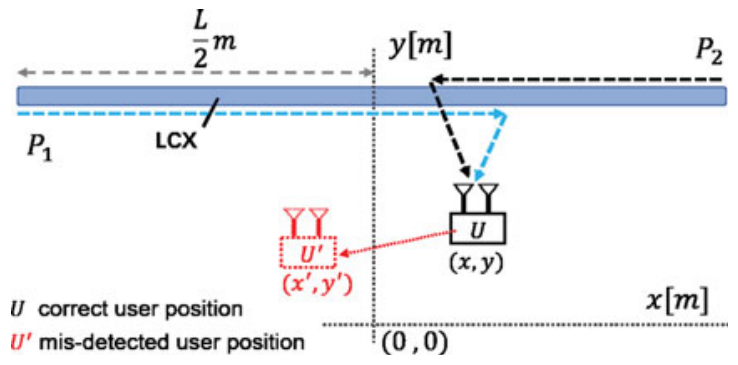

Fig. 4. The power allocation method with user location.

coverage of the LCX power is allocated to each port according to the ratio of the distance between the user and both ends of the cable. The equation of the method in 2-by-2 LCX-MIMO can be given by

$$
\begin{gathered}
P=P_{1}+P_{2}, \\
\frac{P_{1}}{L / 2+x}=\frac{P_{2}}{L / 2-x},
\end{gathered}
$$

where $P$ is the total power, $L$ is the length of the cable. $x$ is the $X$-axis coordinate of the user.

On the other hand, it is impossible to obtain the perfect location of the user and usually localization error will occur due to many factors such as localization algorithm error and noise in the process, etc. As the Fig. 4 shows, $U^{\prime}$ is the mis-detected user position due to localization error and channel capacity can be worse with the power allocation method using the wrong PI $\left(x^{\prime}, y^{\prime}\right)$. This paper will also investigate the capacity performance of LCX-MIMO with our proposed positioning method.

\section{EXPERIMENT AND RESULTS}

\section{A) Experiment configuration}

In the experiment, we measured the channel matrix between the monopole antennas (receiver) and the LCXs (transmitter) with a multi-port vector network analyzer (VNA) in an anechoic chamber. The measurement frequency was set at $2.4 \mathrm{GHz}$ band. As Fig. 5 shows, we place two LCXs perpendicular to each other in the experiment environment and define the intersection as the origin point of the coordinate. LCXs are set with over $1 \mathrm{~m}$ height from the ground of the anechoic chamber. We set 25 measurement points in the wireless coverage area with the $X$-axis range from 1 to $3 \mathrm{~m}$ and the $Y$-axis range from 1 to $3 \mathrm{~m}$. The equal interval between the measurement points is set as $0.5 \mathrm{~m}$. All the measurement points are set at the same height as the LCX. Receiving antennas will be placed at these points and LCX system will detect the location of the receiver terminal with the estimation of signal arrival time using MUSIC method. The distance between the two adjacent monopole antennas was set as $6 \mathrm{~cm}$, which is half wavelength of the signal for $2.4 \mathrm{GHz}$ band. Antenna gain of conventional monopole antenna is $1 \mathrm{dBi}$. Voltage Standing Wave Ratio (VSWR) is 1.1 for both LCX. 


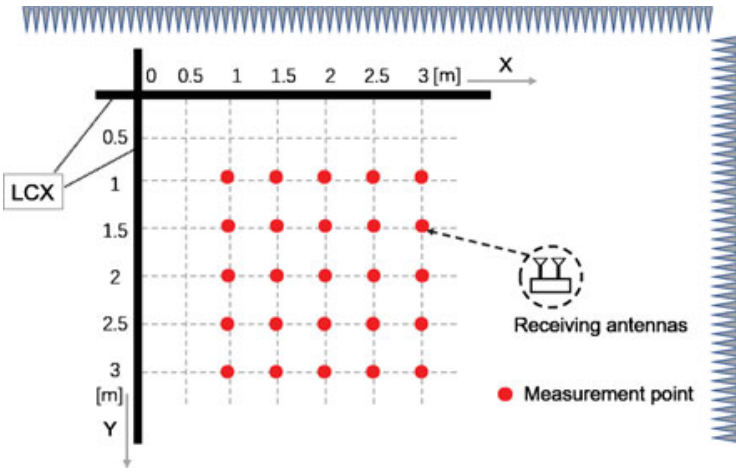

Fig. 5. Experiment configuration.

Table 1. Experimental specifications

\begin{tabular}{ll}
\hline LCX cable type & V-type for both \\
Shortening coefficient $v_{1}, v_{2}$ & 0.6403 for both \\
Maximum radiation angle [deg] & 25 for both \\
Cable loss $[\mathrm{dB} / \mathrm{m}]$ & 0.4 for both \\
Coupling loss $[\mathrm{dB}]$ & $60 \pm 5$ at $1.5 \mathrm{~m}$ \\
Cable diameter [mm] & 7 for both \\
Frequency range [MHz] & $2403.9-2420.1$ \\
& $2403.9-2440.15$ \\
Frequency bandwidth $B[\mathrm{MHz}]$ & $2403.9-2480.15$ \\
Frequency interval [kHz] & $16.25,36.25,76.25$ \\
Number of noise space elements $N$ & 312.5 \\
Time resolution in MUSIC $T_{r}[\mathrm{~ns}]$ & $52,116,244$ \\
\end{tabular}

As the time resolution of the estimation in equation (15), $T_{r}$ can be set to infinitesimal. However, the value of $T_{r}$ has a limitation in real-time estimation, and we should set the value considering the actually measured MUSIC spectrum. For comparison, we set three values for $T_{r}$ as 5, 0.5, 0.1 ns. $N_{s}$ in equation (15) will be set as a suitable value to ensure that the signals arrive within the maximum observation time. We evaluated the localization accuracy over three frequency bands, and Table 1 shows the specifications of the experiment. $N$ is the number of noise space elements which depends on the number of measured samples in frequency domain.

For channel capacity performance evaluation, we calculate the capacity of our 2-by-2 LCX-MIMO using user's location under three conditions that the user location is perfect, user location is detected by single LCX, and user location is detected by our proposed method. For comparison, the capacity results with EP method and WF method will be also provided.

\section{B) Experimental results}

We calculate the direct distance from the user correct position to the detected user position as the localization error. Figure 6 shows the comparison result of the localization error with conventional method and proposed method. Frequency range is from 2403.9 to $2420.1 \mathrm{MHz}$ with bandwidth as $16.25 \mathrm{MHz}$, Time resolution $T_{r}$ is set as $0.5 \mathrm{~ns}$. From the result, our proposal performs better than the conventional method, the maximum error of proposed method is with 1.5

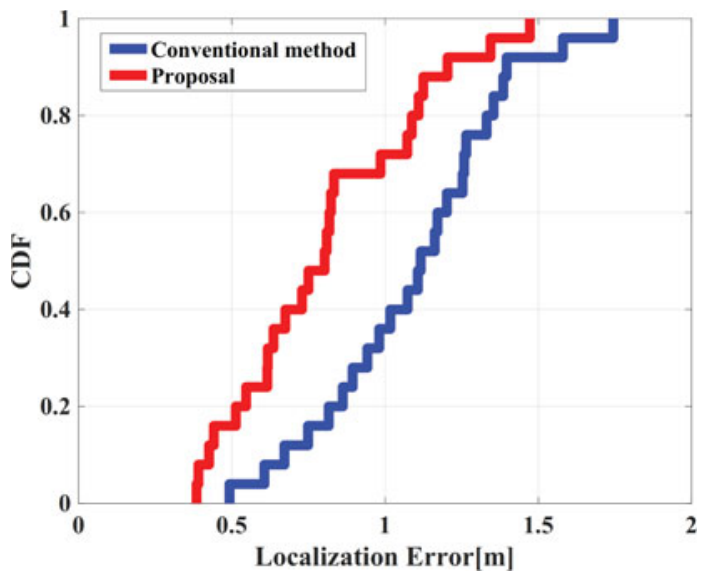

Fig. 6. Results of LCX localization accuracy $(B=16.25 \mathrm{MHz}, \quad N=52$, $\left.T_{r}=0.5 \mathrm{~ns}\right)$.

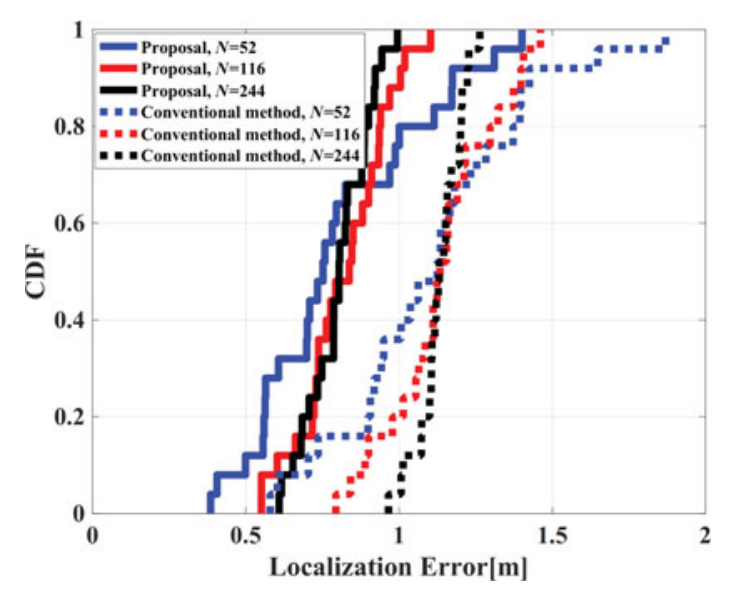

Fig. 7. Results of localization accuracy with different $N$.

$\mathrm{m}$, the minimum error is within $0.5 \mathrm{~m}$. In addition, we take the average value of all errors as the localization accuracy. Our proposed method has the accuracy as about $0.8 \mathrm{~m}$ and the conventional method is $1.15 \mathrm{~m}$.

Figure 7 is the results of Localization accuracy with different $N$ values. When $N$ is 52, the minimum error is small, but the maximum error is large. As $N$ increases, the range of error distribution shrinks. When $N$ is 244 , all measured localization errors with proposed method are within $1 \mathrm{~m}$ and the minimum error is about $0.6 \mathrm{~m}$. We also provide the result of the conventional method for comparison, and our proposed method is better than conventional method in general.

Figure 8 shows the results of the localization error with different time resolution $T_{r}$. It can be found that large value of $T_{r}$ will reduce the localization accuracy as the spatial resolution becomes worse. On the other hand, when $T_{r}$ is set as small as $0.1 \mathrm{~ns}$, the localization accuracy is not much improved than the result with $T_{r}$ as $0.5 \mathrm{~ns}$.

Figures 9 and 10 are the channel capacity results of 2-by-2 LCX-MIMO over $2.4 \mathrm{GHz}$ frequency band. The SNR is set as 6 and $10 \mathrm{~dB}$. The PI method has promising capacity when using proposed positioning method and is better than that using conventional method. In addition, LCX system with 


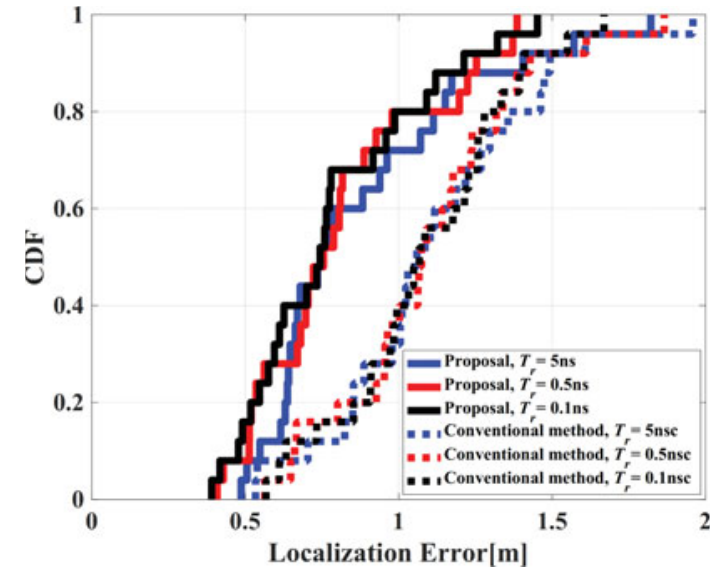

Fig. 8. Results of localization accuracy with different time resolution $T_{r}$.

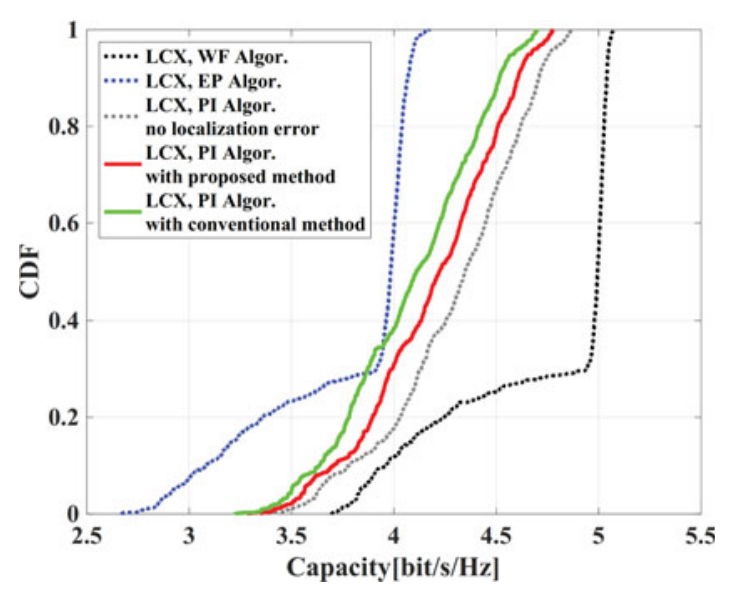

Fig. 9. $\mathrm{CDF}$ of the capacity results $(2.4 \mathrm{GHz}$ band, $S N R=6 \mathrm{~dB})$.

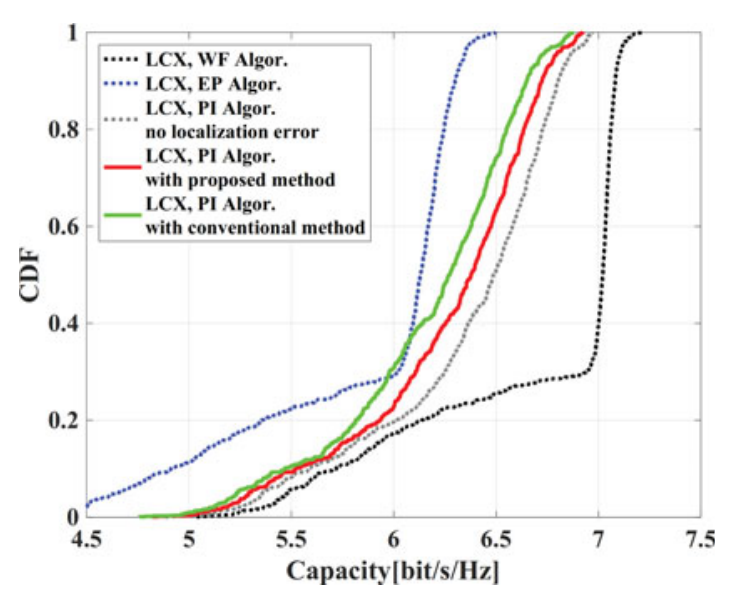

Fig. 10. CDF of the capacity results $(2 \cdot 4 \mathrm{GHz}$ band, $S N R=10 \mathrm{~dB})$.

proposed PI method can have a good capcaity even with localization errors.

We did these measurements in the anechoic chamber as the experimental environment, and this will make the accuracy of the ToA estimation higher. In real environment, interference from noise and other radio wave may deteriorate the ToA estimation. For higher localization accuracy in real environment, we can measure multiple times and take the average of the distribution of the measurement results to reduce the estimation error. In addition, we can use more than two LCX cables with different length and radiation angles, it can also improve the localization accuracy.

\section{CONCLUSION}

In this paper, we propose a LCX positioning method using two LCXs perpendicular to each other. Compared with conventional method, the proposal adds an additional LCX to make a compensation for the lack of detection accuracy of single LCX in the vertical direction. The results show that the positioning performance integrating both location information from two LCXs perpendicular to each other is better than that of conventional method. In addition, we also evaluate the channel capacity of the LCX-MIMO with three power allocation methods. The capacity using PI method using our proposed positioning method is promising.

As the future researches, we will evaluate the localization performance in real environment and optimize the localization algorithm to reduce errors. In this paper, the measurements were done over $2.4 \mathrm{GHz}$ band. We think that the frequency band also gives effects on the performance of the LCX. We will also evaluate the localization accuracy over different frequency bands as our future works.

\section{ACKNOWLEDGMENTS}

This work was supported by the JSPS KAKENHI Grand Number 20Ko4484.

\section{REFERENCES}

[1] Militano, L.; Araniti, G.; Condoluci, M.; Farris, I.; Iera, A.: Deviceto-device communications for $5 \mathrm{G}$ Internet of Things. EAI Endorsed Trans. Internet Things, 15 (1) (2015), 1-15.

[2] Kuutti, S.; Fallah, S.; Katsaros, K.; Dianati, M.; Mccullough, F.; Mouzakitis, A.: A survey of the state-of-the-art localization techniques and their potentials for autonomous vehicle applications. IEEE Internet Things J., 5 (2) (2018), 829-846.

[3] Baker, C.R.: Wireless sensor networks for home health care, in Proc. 21st Int. Conf. Advanced Information Networking and Application Workshops, vol. 2, May 2007, 832-837.

[4] Ojha, T.; Misra, S.; Raghuwanshi, N.S.: Wireless sensor networks for agriculture: the state-of-the-art in practice and future challenges. Comput. Electron. Agric., 118 (2015), 66-84.

[5] Arampatzis, T.H.; Lygeros, J.; Manesis, S.: A survey of applications of wireless sensors and wireless sensor networks, in Proc. 13th Mediterranean Conf: Control Automation, Limassol, Cyprus, June 2005, 719-724.

[6] Yassin, A. et al.: Recent advances in indoor localization: a survey on theoretical approaches and applications. IEEE Commun., Surv. Tutor., 19 (2) (2nd Quart. 2017), 1327-1346.

[7] Wu, C.S.; Yang, Z.; Xiao, C.: Automatic radio map adaptation for indoor localization using smartphones. IEEE Trans.: Mob. Comput., 17 (3) (2018), 517-528. 
[8] Elnahrawy, E.; Austen-Francisco, J.; Ma, R.P.: Adding angle of arrival modality to basic RSS location management techniques, in Proc. 2nd Int. Symp.: Wireless Pervasive Computing, San Juan, Puerto Rico, February 2007, 464-496.

[9] Guvenc, I.; Chong, C.: A survey on TOA based wireless localization and NLOS mitigation techniques. IEEE Commun., Surv. Tutor., 11 (3) (3rd Quarter 2009), 107-124.

[10] Mazuelas, S. et al.: Robust indoor positioning provided by real-time RSSI values in unmodified WLAN networks. IEEE J. Sel.Top. Signal Process., 3 (5) (2009), 821-831.

[11] Pathak, P.H.; Feng, X.; Hu, P.; Mohapatra, P.: Visible light communication, networking, and sensing: a survey, potential and challenges. IEEE Commun., Surv. Tutor., 17 (4) (4th Quart., 2015), 2047-2077.

[12] Hauschildt, D.; Kirchhof, N.: Advances in thermal infrared localization: challenges and solutions, in Proc. IEEE Int. Conf: Indoor Positioning Indoor Navigation (IPIN), September 2010, 1-8.

[13] Hazas, M.; Hopper, A.: Broadband ultrasonic location systems for improved indoor positioning. IEEE Trans. Mob. Comput., 5 (5) (2006), 536-547.

[14] Santo, H.; Maekawa, T.; Matsushita, Y.: Device-free and privacy preserving indoor positioning using infrared retro-reflection imaging, in Proc. IEEE Int. Conf:: Pervasive Computing and Communications, Kona, HI, USA, March 2017, 52-141.

[15] Nakamura, N.; Tsunomachi, H.; Fukui, R.: Road vehicle communication system for vehicle control using leaky coaxial cable. IEEE Commun. Mag., 34 (10) (1996), 84-89.

[16] Okamoto, T.; Duong, Q.T.; Higashino, T.; Okada, M.: A proposal of data transmission in parallel line fed wireless power transfer, 2015 15th International Symposium on Communications and Information Technologies (ISCIT), 2015, 85-88.

[17] Fokum, D.T.; Frost, V.S.: A survey on methods for broadband internet access on trains. IEEE Commun., Surv. Tutor., 12 (2) (Second Quarter 2010), 171-185.

[18] Suzuki, F.; Niwa, A.; Takano, K.: Thin leaky coaxial cable LCX-5D, in Proc. IEICE General Conf. 2012, March 2012, B-1-156-837.

[19] Dudley, D.; Lienard, M.; Mahmoud, S.; Degauque, P.: Wireless propagation in tunnels. IEEE Antennas Propag. Mag., 49 (2) (2007), 11-26.

[20] Nishikawa, K.; Higashino, T.; Tsukamoto, K.; Komaki, S.: A new position detection method using leaky coaxial cable. IEICE Electron. Express, 5 (8) (2008), 285-290.

[21] Hou, Y.; Tsukamoto, S.; Ariyoshi, M.; Kobayashi, K.; Kumagai, T.; Okada, M.: Performance comparison for 2 by 2 MIMO system using single leaky coaxial cable over WLAN frequency band, in Proc. 25th Asia-Pacific Signal and Information Processing Association Annual Summit and Conference (APSIPA ASC 2014), December 2014, 1-6.

[22] Hou, Y.; Tsukamoto, S.; Li, S.; Higashino, T.; Kobayashi, K.; Okada, M.: Capacity evaluation of MIMO channel with one leaky coaxial cable used as two antennas over linear-cell environments. IEEE Trans. Veh. Technol., 66 (6) (2017), 4636-4646.

[23] Tsukamoto, S.; Maeda, T.; Ariyoshi, M.; Hou, Y.; Kobayashi, K.; Kumagai, T.: An experimental evaluation of $2 \times 2$ MIMO system using closely-spaced leaky coaxial cables, in Proc. 25th Asia-Pacific Signal and Information Processing Association Annual Summit and Conference (APSIPA ASC 2014), December 2014, 1-6.

[24] Hou, Y.et al.: Configuration of MIMO system using single leaky coaxial cable for linear cell environments. IEICE Commun. Express, 4 (5) (2015), 143-148.

[25] Hou, Y.; Tsukamoto, S.; Ariyoshi, M.; Kobayashi, K.; Okada, M.: 4-by4 MIMO channel using two leaky coaxial cables (LCXs) for wireless applications over linear-cell, in Proc. 3 rd IEEE Global: Conference on Consumer Electronics (GCCE 2014), October 2014, 125-126.
[26] Higashino, T.; Okada, M.; Maeda, T.; Tsukamoto, S.: An evaluation of error performance of position location in the LCX liner cell MIMO system, in Proc. Int. Technical Conference on Circuits/Systems Computers Communication, July 2014, 749-751.

[27] Oki, S.; Hou, Y.; Higashino, T.; Okada, M.: Two-dimensional positioning for radio terminal in 4-by-4 MIMO system using leaky coaxial cable antenna. ITE Techn. Rep., 39 (4) (2015), 45-48.

[28] Guan, Q.; Chen, C.; He, C.: A novel sensor using VHF zigzag-slotted leaky coaxial cable for intruder localization. Microw. Opt. Technol. Lett., 60 (2018), 634.

[29] Liu, Y.; Shi, Y.; Guo, J.; Wang, Y.: Application of pulse compression technique in fault detection and localization of leaky coaxial cable. IEEE Access, 6 (2018), 66709-66714.

[30] $\mathrm{Xu}, \mathrm{H}$. et al.: A highresolution leaky coaxial cable sensor using a wideband chaotic signal. Sensors, 18 (12) (2018), 4154.

[31] Qiao, J.; Xu, H.; Zhang, J.; Han, H.; Wang, B.: High-resolution and anti-jamming chaotic guided radar prototype for perimeter intrusion detection. J. Electromagn. Waves Appl., 33 (8) (2019), 1060-1069.

[32] Hou, Y.; Zhu, J.; Denno, S.; Okada, M.: Capacity of 4-by-4 MIMO channel using one composite leaky coaxial cable with user position information. IEEE Trans. Veh. Technol., 33 (8) (2019), 10601069.

[33] Goldsmith, A.; Jafar, S.; Jindal, N.; Vishwanath, S.: Capacity limits of MIMO channels. IEEE J. Sel. Area Commun., 21 (5) (2003), 684-702.

[34] Khalighi, M.; Brossier, J.; Jourdain, G.; Raoof, K.: Water filling capacity of Rayleigh MIMO channels, in Proc. 12th IEEE international Symposium on Personal, Indoor and Mobile Radio Communications (PIMRC 2001), September 2001, A. 115-118.

Junjie Zhu was born in Jiangsu, China. He received the B.E. degree in communication engineering from Nanjing University of Information Science and Technology, Nanjing, China, in 2016. He received the M.E. degree and is studying for Ph.D. from the Department of Natural Science and Technology, Okayama University, Japan. His research interests include wireless communication and signal processing.

Pengcheng Hou was born in Beijing, China. He received the B.E. degree in communication engineering from Hunan University, Changsha, China, in 2019. He is studying for M.E. degree from the Department of Natural Science and Technology, Okayama University, Japan. His research interests include wireless communication and signal processing.

Yafei Hou received his Ph.D. degrees from Fudan University, China and Kochi University of Technology (KUT), Japan in 2007. He was a post-doctoral research fellow at Ryukoku University, Japan from August 2007 to September 2010. He was a research scientist at Wave Engineering Laboratories, ATR Institute International, Japan from October 2010 to March 2014. He was an Assistant Professor at the Graduate School of Information Science, Nara Institute of Science and Technology, Japan from April 2014 to March 2017. He became an Assistant Professor at the Graduate School of Natural Science and Technology, Okayama University, Japan from April 2017. $\mathrm{He}$ is a guest research scientist at Wave Engineering Laboratories, ATR Institute International, Japan from October 2016. His research interests are communication systems, wireless networks, and signal processing. He received IEICE (the Institute of Electronics, Information and Communication Engineers) 
Communications Society Best Paper Awards in 2016, 2020 and Best Tutorial Paper Award in 2017. Dr. Hou is a senior member of IEEE and member of IEICE.

Satoshi Denno received the M.E. and Ph.D. degrees from Kyoto University, Kyoto, Japan in 1988 and 2000, respectively. He joined NTT radio communications systems labs, Yokosuka, Japan, in 1988. In 1997, he was seconded to ATR adaptive communications research laboratories, Kyoto, Japan. From 2000 to 2002, he worked for NTT DoCoMo, Yokosuka, Japan. In 2002, he moved to DoCoMo communications laboratories Europe $\mathrm{GmbH}$, Germany. From 2004 to 2011, he worked as an associate professor at Kyoto University. Since 2011, he is a full professor at graduate school of natural science and technology, Okayama University. From the beginning of his research career, he has been engaged in the research and development of digital mobile radio communications. In particular, he has considerable interests in channel equalization, array signal processing, Space time codes, spatial multiplexing, and multimode reception. He received the Excellent Paper Award from the IEICE in
1995 and IEICE Communications Society Best Paper Awards in 2020.

Minoru Okada received the B.E. degree in communications engineering from the University of Electro-Communications, Tokyo, Japan, in 1990, and the M.E. and Ph.D. degrees in communications engineering from Osaka University, Osaka, Japan, in 1992 and 1998, respectively, where he was a Research Associate, from 1993 to 2000 . From 1999 to 2000 , he was a Visiting Research Fellow with the University of Southampton, Southampton, UK. In 200o, he joined the Graduate School of Information Science, Nara Institute of Science and Technology, Ikoma, Japan, as an Associate Professor and then became a Professor. His current research interests include wireless communications, including WLAN, WiMAX, code division multiple access, OFDM, and satellite communications.,Dr. Okada is a member of the Institute of Television Engineers of Japan, Institute of Electrical, Information, and Communication Engineers (IEICE), Japan, and the Information Processing Society Japan. He was a recipient of the Young Engineer Award from the IEICE, in 1999. 